\title{
Fever and neutropenia hospital discharges in children with cancer: A 2012 update
}

Emily L Mueller MD, MSc ${ }^{1,2}$

James Croop, MD, $\mathrm{PhD}^{1}$

Aaron E Carroll, MD, $\mathrm{MS}^{2,3}$

1. Section of Pediatric Hematology Oncology, Department of Pediatrics, Indiana University, Indianapolis, IN 46202

2. Pediatric and Adolescent Comparative Effectiveness Research, Indiana University, Indianapolis, IN 46202

3. Center for Health Policy and Professionalism Research, Indiana University, Indianapolis, IN 46202

Correspondence:

Emily Mueller, MD, MSc

Indiana University School of Medicine

410 West 10th Street, Suite 4099C

Indianapolis, IN 46202

Cell: 312-399-0245 Fax: 317-321-0128

elmuelle@iu.edu

Abstract word count: 249

Text word count: 3043

Short Running Head: National Discharges for Peds Ca FN Update

Key words: Child, Adolescent, Oncology, Supportive Care, Febrile Neutropenia, Health Care Surveys, United States

Number of tables: 4

Number of figures: 0

Supplemental files: 1

Abbreviation table:

\begin{tabular}{|l|l|}
\hline AHRQ & Agency for Healthcare Research and Quality \\
\hline ALL & acute lymphoblastic leukemia \\
\hline CCS & Clinical Classification Software \\
\hline FN & Fever and neutropenia \\
\hline HCUP & Healthcare Cost and Utilization Project \\
\hline ICD-9-CM & $\begin{array}{l}\text { International Classification of Diseases, Ninth Revision, Clinical } \\
\text { Modification }\end{array}$ \\
\hline KID & Kids' Inpatient Database \\
\hline LOS & Length of stay \\
\hline SLOS & "Short length of stay" \\
\hline US & United States \\
\hline
\end{tabular}

This is the author's manuscript of the article published in final edited form as: Mueller, E. L., Croop, J., \& Carroll, A. E. (2016). Fever and neutropenia hospital discharges in children with cancer: A 2012 update. Pediatric Hematology and Oncology, 33(1), 39-48. http://doi.org/10.3109/08880018.2015.1102998 


\begin{abstract}
BACKGROUND: Fever and neutropenia (FN) is a common precipitant for hospitalization among children with cancer, but hospital utilization trends are not well described. This study describes national trends for hospital discharges for FN among children with cancer for the year 2012, compared to our previous analysis from 2009.
\end{abstract}

METHODS: Data were analyzed from the Kids' Inpatient Database (KID), an all-payer US hospital database, for 2012. Pediatric patients with cancer who had a discharge for FN were identified using: age $\leq 19$ years, urgent or emergent admit type, non-transferred, and a combination of ICD-9-CM codes for fever and neutropenia. We evaluated factors associated with a "short length of stay" (SLOS). Sampling weights were used to permit national inferences.

RESULTS: In 2012, children with cancer accounted for $1.8 \%$ of pediatric hospital discharges $(n=120,675)$, with $12.2 \%(n=13,456)$ of cancer-related discharges meeting FN criteria. Two-fifths of FN discharges had a SLOS, which accounted for \$91 million (2015 US\$) in hospital charges. The majority had no serious infections; most common infections were viral infection $(9.6 \%)$ or upper respiratory infection $(9.6 \%)$. Factors significantly associated with SLOS included having a diagnosis of ear infection $(\mathrm{OR}=1.54$, CI 1.16-2.03), soft tissue sarcoma (OR=1.47, CI 1.10-1.95), and Hodgkin lymphoma (OR 1.51, CI 1.09-2.10), as compared with not having those diagnoses.

CONCLUSION: SLOS admissions continue to be rarely associated with serious infections, but contribute substantially to the burden of hospitalization for pediatric FN. Implementation of risk stratification schemas to identify patients who meet low risk criteria may decrease financial burden. 


\section{Introduction}

Among pediatric patients with cancer, substantial research exists related to the risk stratification, prevention and treatment of fever and neutropenia (FN).[1] FN continues to be a common and potentially life-threatening complication of chemotherapy[2,3], requiring urgent evaluation and hospitalization.[4,5] Evidence shows that some pediatric patients with cancer who develop FN could be treated as outpatients or have an early discharge or even be treated as an outpatient.[6-8] Adoption of these practices would require institution specific systems to be in place for risk stratification and outpatient monitoring, with consideration for patient travel and social circumstances.[9]

Evaluation of the impact of FN on health care utilization in the United States (US) could lead to improved insight into both hospital- and patient-level factors that impact length of stay. In our previous study of pediatric patients with cancer who were discharged from the hospital with FN, we examined factors associated with a "short length of stay" (SLOS) of 3 days or less. We analyzed a nationally representative database, the Healthcare Cost and Utilization Project Kids Inpatient Database for 2009, and found that SLOS discharges contributed substantially to the burden of hospitalization for pediatric FN, but were rarely associated with serious infections. This supports the need for continued evaluation of the financial and resource usage by hospital systems, insurance payers, and the patients and families these hospitalizations impact.

The objective of this study was to further characterize discharges for children with cancer who are admitted non-electively and discharged with a diagnosis of fever and neutropenia, across the United States, utilizing the most recent pediatric hospitalization 
data from 2012. Understanding the national patterns of health care usage for pediatric patients with FN could provide insight into national adoption of alternative management practices for FN among children with cancer. 


\section{Materials and Methods}

\section{Study Design and Setting}

We identified pediatric patients with cancer admitted for FN from a crosssectional analysis of pediatric discharges in 2012, using the Healthcare Cost and Utilization Project's (HCUP) Kids' Inpatient Database (KID), compiled by the Agency for Healthcare Research and Quality (AHRQ).[10] The KID is a nationally representative database that samples $80 \%$ of pediatric discharges and $10 \%$ of uncomplicated births from both teaching and non-teaching hospitals. This dataset has been compiled every third year since 1997. Our original analysis utilized data from the 2009 KID.[11] For the current analysis we utilized data from the most recently available calendar year (2012).

The KID is designed to provide the statistical power to detect and evaluate rare conditions among hospitalized children. Multiple publications have utilized the KID to examine hospitalization patterns of care for children in the US.[12-16] The KID include data elements such as International Classification of Diseases, Ninth Revision, Clinical Modification (ICD-9-CM) codes, length of stay (LOS), patient demographic characteristics, and hospital charges per episode of care.

In an effort to facilitate research utilizing ICD-9-CM codes, HCUP developed the Clinical Classification Software (CCS), which can groups thousands of ICD-9-CM codes into 260 mutually exclusive diagnostic groups.(Elixhauser). Several other publications have utilized CCS codes to analyze administrative data specific to children with cancer.(Mueller NEDS, Mueller KID, Russell H, Goudie Pediatrics) It is imperative to recognize that administrative data is presumed to be the diagnoses provided by clinical 
providers or staff. For this manuscript, CCS codes are utilized for the grouping of patients by type of cancer and to identify co-occuring infections (See Supplemental).

\section{Identification of Cases}

In keeping with our earlier analysis, we determined hospitalizations for pediatric patients with cancer using the HCUP CCS codes. Pediatric patients with cancer were defined as those encounters having a CCS codes between 11-45, which encompass all types of malignancies. We chose to exclude transfers from all analyses in order to assess LOS accurately and to avoid duplicate encounters for the same episode of care (since patient level identifiers are not available in this dataset). Patients who died were included in our analyses.

\section{Outcome and Explanatory Variables}

As was done in our previous analysis, we chose to capture patients who were admitted due to the presence of fever in the setting of neutropenia, rather than those who may have had a fever during the course of their inpatient stay for chemotherapy. This decision was made in order to focus on the population of patients where outpatient management could have been a consideration. We defined a discharge encounter for "fever and neutropenia" (FN) using the following criteria: age $\leq 19$ years, admit type either urgent or emergent (i.e., excluded elective admissions), and a combination of ICD9-CM discharge diagnosis of fever $(780.6, .60, .61)$ and "neutropenia" (neutropenia [288.0], pancytopenia [284.1], or decreased white blood cell count [112.5]) in any of the discharge diagnosis fields.

Co-occurring infections were evaluated by ranking the frequency of all CCS codes for infections. We then included the 10 most common CCS infectious diagnoses 
(see Supplemental table) as separate variables in our analyses: septicemia (CCS code 2), viral infection (7), bacterial infection (3), fungal infection (4), upper respiratory infection (126), intestinal infection (135), pneumonia (122), skin infection (197), urinary tract infection (159), and ear infection (92).

The primary outcomes of interest were factors associated with discharges that had SLOS of 3 days or less. The duration of three days was chosen in order to evaluate patients whose hospital stay would have been approximately 48 hours, since hospital days are counted when they cross midnight. Therefore a patient admitted later in the day could spend only 48 hours in the hospital, but their LOS would be counted as 3 days.

Secondary outcomes for FN discharges, derived from variables available in the KID, included population-adjusted frequency of discharges, average LOS, proportion of encounters with infectious diagnoses, and mean and total charges by length of stay categories.

\section{Data Analyses}

Discharges within the KID are weighted based on the sampling scheme in order to permit inferences for a nationally representative population. HCUP provides supplementary sampling and weighting details.[10] Statistical analyses were performed using STATA version 13.0 (Stata Corp, College Station, Texas). Survey estimation commands (SVY commands) in STATA were used to incorporate the KID sample design variables. The following variables were used for survey estimation: "HOSPID” for cluster, "KID_STRATUM" for stratum and "DISCWT" for sample weight. All data are presented as weighted values to represent the nationally representative population, unless specified otherwise. The 2012 KID data contains over 3 million unweighted discharges 
that, after application of the survey weights, represented over 6.5 million weighted discharges in the population. Our analysis was based on de-identified national data and was therefore considered exempt from human subjects review by the Indiana University Medical Institutional Review Board.

We utilized descriptive statistics to determine the characteristics of FN discharges by gender, age, race/ethnicity, and primary payer type, hospital teaching and location status, and hospital Census region. The frequency of FN discharges was described as the rate of discharges per 100,000 US children per year, using national Census data for 2012.[17] Census data were chosen as the denominator due to the fact that prevalence of pediatric patients with cancer is not well described. Hospital charges were recalculated from 2012 US dollar (\$) to 2015 US dollar using the Consumer Price Index.[18]

A weighted multivariate logistic regression model, utilizing the HCUP provided weights to produce nationally representative results, was performed to estimate factors associated with a SLOS among pediatric oncology encounters for FN; statistical weights were used to account for clustering of patients by hospital. The explanatory variables for the regression model were selected a priori based on hypothesized clinical relevance and were consistent with our previous analysis, which included: patient's age, gender, primary expected payer, median household income for the patient's zip code, hospital location and teaching status, hospital Census region, dichotomous variables for the presence or absence of each of the top 10 most common cancer diagnoses, and dichotomous variables for types of infections. We hypothesized that hospital level and sociodemographic factors impact the decision-making regarding length of stay for 
patients hospitalized with FN. Statistically significant associations were determined if pvalue was less than 0.05 . 


\section{Results}

Characteristics of the study population

In 2012, there were a total of $6,675,222$ weighted US pediatric discharges in the KID. Within those, $1.8 \%(n=120,675)$ had a diagnosis of cancer. According to the HCUP classification system, $54.5 \%(n=60,241)$ of pediatric cancer patient hospitalizations had non-elective admissions (coded as either emergent or urgent) that did not include a transfer. Among non-transferred discharges for pediatric patients with cancer, $12.2 \%$ $(n=13,456)$ met the criteria for $\mathrm{FN}$; this accounted for $22.3 \%$ of all non-elective, nontransferred pediatric cancer discharges. Only $0.9 \%(n=1,015)$ of non-transferred inpatient discharges for pediatric patients with cancer ended in death, and only $65(0.5 \%)$ that were pediatric FN discharges.

The characteristics of the discharges for pediatric patients with cancer that met FN criteria are presented in Table I. The majority of patients with FN discharges were between the ages of 0-9 years, which was higher than the proportion among overall pediatric cancer discharges for patients between 0-9 years of age. The population of FN discharges were predominantly male and non-Hispanic white race. Most FN encounters had insurance coverage, with private greater than public insurance. Additionally, the proportion of patient discharges with a diagnosis of acute lymphoblastic leukemia (ALL) was higher among the FN discharges $(43.6 \%)$ compared to the overall sample $(26.9 \%)$. In 2012, population-adjusted $\mathrm{FN}$ discharges among all pediatric patients with cancer occurred at a rate of 16.3 per 100,000 US children. During 2012, FN discharges for pediatric patients with cancer represented 100,845 total inpatient days (compared with 823,868 total days for all pediatric cancer discharges). 


\section{Discharge characteristics for pediatric FN discharges}

The average LOS for FN discharges was 7.5 days, and 39\% of discharges had a SLOS $(n=5,230)$. The most common infections among those with a SLOS were viral infection $(9.6 \%)$ or upper respiratory infection $(9.6 \%)$, but $66.4 \%$ had no infection identified. Conversely for patient discharges with a LOS greater than 3 days, the most commonly associated infections were septicemia (15.5\%), viral infection (12.1\%), bacterial infection (11.4\%), and fungal infection (9.2\%) (Table II).

\section{Charges for pediatric FN discharges}

The mean hospital charge was $\$ 65,536$ for FN discharges among pediatric patients with cancer, with the total charges of about $\$ 881$ million (Table III). The mean charges varied by LOS category with the average charge for a SLOS being $\$ 17,437$ versus greater than 3 days was $\$ 96,023$. There was a difference of $\$ 1,261$ between the mean daily charges for those patients with a SLOS versus those with a LOS greater than 3 days. SLOS discharges accounted for $\$ 91.2$ million in charges for 2012 .

Factors associated with a SLOS for pediatric FN discharges

In the multivariate analysis (Table IV), factors associated with significantly increased odds of a SLOS for FN included having a diagnosis of an ear infection $(\mathrm{OR}=1.54, \mathrm{CI} 1.16-2.03)$, soft tissue sarcoma $(\mathrm{OR}=1.47$, CI 1.10-1.95), and Hodgkin lymphoma $(\mathrm{OR}=1.51$, CI 1.09-2.10) as compared with not having those diagnoses. Conversely, socio-demographic factors associated with not having a SLOS included nonwhite race $(\mathrm{Black} \mathrm{OR}=0.78, \mathrm{CI} 0.65-0.94$; Hispanic $\mathrm{OR}=0.85, \mathrm{CI} 0.72-0.99)$ versus white race, and having Healthcare Cost and Utilization Project designated “Other” payer $(\mathrm{OR}=0.74, \mathrm{CI} 0.59-0.94)$ versus private insurance. 


\section{Discussion}

This study provides the most recent nationally representative analysis of inpatient hospital discharges for FN among pediatric patients with cancer in the US. Overall, among non-elective and non-transferred discharges, we found that in both 2009 and 2012 about 1 of every 5 pediatric cancer-associated hospital discharges are related to FN. Among FN discharges, in both years two-fifths had a SLOS; yet hospital charges were up from \$65.5 million in 2009 to \$91.2 million in 2012 (expressed in 2015 US\$). An increased odds of having a SLOS in 2012 were found among patients with ear infections, soft tissue sarcoma or Hodgkin lymphoma. Conversely, non-white race and "Other payer" were found to have decreased odds of a SLOS. This data emphasizes the continued need to investigate alternate methods of caring for children with cancer who experience FN, especially for those without serious infections.

FN remains a common reason for hospitalization among children with cancer accounting for $12.2 \%$ of discharges, up from $10.1 \%$ in 2009 .[11] Similarly, the rate of FN discharges among the US population (age-adjusted) increased from 13.4 to 16.3 per 100,000 US children. The total number of inpatient days has increased dramatically from 79,870 to 100,845 as well. This increase cannot be fully explained by changes in the incidence or improvements in treatment intensity and cure rates over this relatively short time frame based on most recent statistics.[19] A significant body of literature supports risk stratification schemas to decrease hospitalization for FN and evidence supports the safety and efficacy of outpatient management[6-8], yet these do not seem to be translating into less hospitalizations for this population based on this nationally representative analysis. 
The proportion of patients with a SLOS among all FN discharges has remained relatively steady, 39\% in 2012 from $41 \%$ in 2009 . For all FN discharges, the mean LOS has gone up slightly from 7.1 days in 2009 to 7.5 days in 2012, contrary to the previously published multi-institution study by Basu, et al that had found a 3\% decrease in LOS for FN per year.[20]

This database provides a unique way in which to monitor the hospital trends for children with cancer who experience febrile neutropenia. If risk stratification schemas are implemented nationally, we should expect to see a decrease in the proportion of FN admissions among children with cancer as those who meet low risk criteria are beginning to receive outpatient therapy. Similarly, if the step-down approach is implemented nationally (admission then discharge after a short observation period of observation, but prior to count recovery), the proportion of patients with a SLOS should increase. Since the proportion of those who require medical interventions, other than intravenous antibiotics, will remain after 2-3 days. An interesting future evaluation utilizing the KID would be to assess how the associated diagnoses may change once ICD-10-CM codes are implemented, possibly leading to more granular information that can be ascertained from administrative databases.

While we do not believe it is feasible to use discharge diagnoses (ICD-9-CM codes) in order to devise triaging or new management styles, there are several consistent trends in both 2009 and 2012 that should be taken into consideration. In both years analyses, it was found that having an oncologic diagnosis of soft tissue sarcoma or Hodgkin lymphoma was associated with having a SLOS, thus was associated with a very low proportion of serious infection diagnoses. Patients with these diagnoses may 
represent an ideal group for further evaluation and application of risk stratification schemas.

As clinically anticipated, infections were commonly found among discharges for FN, but serious infections remain uncommon among those patients with a SLOS. In both the 2009 and 2012 analyses, ear infections were associated with having a SLOS. This likely represents a population of patients whose source of fever in the setting of neutropenia would be easily identified on careful examination. The question remains as to whether these findings were known prior to admission, which may depend on the comfort level of the provider doing the urgent care assessment. Pediatric providers are likely more comfortable examining the ears of young children and more highly trained in the diagnosis of ear infections. It would be intriguing to have more specific data about FN discharges where ear infections are diagnosed in order to determine if this population represents a low risk group who would be amenable to outpatient management.

Mortality remains low among FN hospitalizations for children with cancer, currently found to be $0.5 \%$ as compared with $0.4 \%$ in 2009 . The mortality rate among this nationally representative population is lower than previously documented.[20] While death will always remain an important outcome to monitor, it will need to be assessed as part of a larger set of outcomes given the very low incidence.[21]

As evidenced by this analysis, the financial burden of FN care continues to climb. The average charges per FN discharge increased 26\% from 2009 to 2012 , with the overall charges for SLOS FN discharges increasing by almost $140 \%$. This trend clearly demonstrates that efforts to decrease the number and length hospitalization for children with FN are necessary from a financial perspective. How children with cancer who 
experience FN interact with the healthcare system is still not fully understood, as few datasets include pertinent patient and family level information regarding distance to treating institution, travel resources and institutional practices that can all impact the ability to treat low risk FN pediatric patients in the outpatient setting. The involvement of all stakeholders will ultimately be necessary to negotiate ways in which safety, efficacy, and cost are all taken into consideration.

While a major strength of this study is to provide a nationally representative overview of FN discharges for children with cancer, it has several important limitations. Administrative data analyses rely on accuracy of ICD-9-CM codes to identify the population and medical issues of interest, which are subject to errors of omission or commission. As described in our previous analysis, the type of admission was inferred based on discharge diagnoses. FN is not a single diagnostic code, nor will it be in ICD10-CM, and was indicated by the presence of both ICD-9-CM codes for fever and neutropenia. It is also still possible that this was an underestimation of patients with FN given that some patients who present with fever and neutropenia may be discharged with only the diagnosis of neutropenia. We documented that the rates of FN discharges increased, but we did not analyze rates of discharges for other pediatric conditions, which may also have increased. We attempted to use the national census to adjust for changes in the population, due to the fact that there are no good data on the prevalence of childhood cancer, rather only incidence. 


\section{Conclusion}

Our updated study demonstrates that the impact of FN discharges among pediatric patients with cancer on healthcare utilization has continued to climb. As compared with our previous analysis, we have demonstrated that despite evidence supporting the feasibility and safety of alternative approaches to clinical management of FN, the burden upon the healthcare system has not improved. The continued documentation of the burden of FN will be key in expanding awareness and increasing motivation for a larger study to improve the care of pediatric patients with cancer experiencing FN. 
Acknowledgements: We would like to thank Achamyeleh Gebremariam from University of Michigan for his methodology expertise and guidance.

Declaration of Interest Statement: All authors have no conflicts of interest to disclose. 


\section{References}

1. Lehrnbecher T, Phillips R, Alexander S, Alvaro F, Carlesse F, Fisher B, Hakim H, Santolaya M, Castagnola E, Davis BL, Dupuis LL, Gibson F, Groll AH, Gaur A, Gupta A, Kebudi R, Petrilli S, Steinbach WJ, Villarroel M, Zaoutis T, Sung L, International Pediatric Fever Neutropenia Guideline Panel. Guideline for the management of fever and neutropenia in children with cancer and/or undergoing hematopoietic stem-cell transplantation. J Clin Oncol. 2012;30:4427-4438.

2. Bodey GP, Buckley M, Sathe YS, Freireich EJ. Quantitative Relationships between circulating leukocytes and infection in patients with acute leukemia.

3. Mullen CA. Which children with fever and neutropenia can be safely treated as outpatients? Br J Haematol. 2001;112:832-837.

4. Pizzo PA. Management of fever in patients with cancer and treatment-induced neutropenia. N Engl J Med. 1993;328:1323-1332.

5. Freifeld AG, Bow EJ, Sepkowitz KA, Boeckh MJ, Ito JI, Mullen CA, Raad, II, Rolston KV, Young JA, Wingard JR, Infectious Diseases Society of America. Clinical practice guideline for the use of antimicrobial agents in neutropenic patients with cancer: 2010 update by the infectious diseases society of america. Clin Infect Dis. 2011;52:e56-93.

6. Manji A, Beyene J, Dupuis LL, Phillips R, Lehrnbecher T, Sung L. Outpatient and oral antibiotic management of low-risk febrile neutropenia are effective in children--a systematic review of prospective trials. Support Care Cancer. 2012;20:1135-1145.

7. Teuffel O, Ethier MC, Alibhai SM, Beyene J, Sung L. Outpatient management of cancer patients with febrile neutropenia: a systematic review and meta-analysis. Ann Oncol. 2011;22:2358-2365.

8. Vidal, L Ben dor I, Paul M, Pokroy E, Soares-Weiser K, Leibovici L. Oral versus intravenous antibiotic treatement for febrile neutropenia. Cochrane Database Syst Rev. 2013;10:CD003992.

9. Henry M, Sung L. Supportive Care in Pediatric Oncology: Oncologic Emergencies and Management of Fever and Neutropenia. Pediatr Clin North Am. 2015;62:27-46.

10. Overview of the Kids' Inpatient Database (KID). Healthcare Cost and Utilization Project (HCUP): 2012. [http://www.hcup-us.ahrq.gov /kidoverview.jsp]

11. Mueller EL, Walkovich KJ, Mody R, Gebremariam A, Davis, MM. Hospital discharges for fever and neutropenia in pediatric cancer patients: United States, 2009. BMC Cancer. 2015;15:388.

12. Byington CL, Wilkes J, Korgenski K, Sheng X. Respiratory Syncytial VirusAssociated Mortality in Hospitalized Infants and Young Children. Pediatrics. 2015;135:e24-31.

13. Odetola FO, Gebremariam A, Freed GL. Patient and hospital correlates of clinical outcomes and resource utilization in severe pediatric sepsis. Pediatrics. 2007;119:487-494.

14. Patrick SW, Schumacher RE, Benneyworth BD, Krans EE, McAllister JM, Davis MM. Neonatal abstinence syndrome and associated health care expenditures: United States, 2000-2009. JAMA. 2012;307:1934-1940. 
15. Setty BA, O'Brien SH, Kerlin BA. Pediatric venous thromboembolism in the United States: a tertiary care complication of chronic diseases. Pediatr Blood Cancer. 2012;59:258-264.

16. Woolford SJ, Gebremariam A, Clark SJ, Davis MM. Persistent gap of incremental charges for obesity as a secondary diagnosis in common pediatric hospitalizations. J Hosp Med. 2009;4:149-156.

22. Annual Estimates of the Resident Population for Selected Age Groups by Sex for the United States, States, Counties, and Puerto Rico Commonwealth and Municipios: April 1, 2010 to July 1, 2012, Source: U.S. Census Bureau, Population Division, Release Date: June 2013

23. Consumer Price Index Inflation Calculator. [http://data.bls.gov/cgi-bin/cpicalc.pl]

24. Ward E, DeSantis C, Robbins A, et al. Childhood and adolescent cancer statistics, 2014. CA Cancer J Clin. 2014:64(2):83-103.

25. Basu SK, Fernandez ID, Fisher SG, Asselin BL, Lyman GH. Length of stay and mortality associated with febrile neutropenia among children with cancer. $J$ Clin Oncol. 2005;23:7958-7966.

26. Haeusler GM, Phillips RS, Lehrnbecher T, Thursky KA, Sung L, Ammann RA. Core outcomes and definitions for pediatric fever and neutropenia research: A consensus statement from an international panel. Pediatr Blood Cancer. 2015;62:483-489. 